\title{
Educación superior y la COVID-19: adaptación metodológica y evaluación online en dos universidades de Barcelona
}

\author{
*Daniel Ortega Ortigoza \\ Facultad de Ciencias de la Educación, Universidad Autónoma de Barcelona, España \\ https://orcid.org/0000-0002-8581-4833 \\ Julio Rodríguez Rodríguez \\ Facultad de Educación, Universidad de Barcelona, España \\ https://orcid.org/0000-0001-9202-2589 \\ Ainoa Mateos Inchaurrondo \\ Facultad de Educación, Universidad de Barcelona, España \\ https://orcid.org/0000-0002-1159-9966
}

Recibido: 31/08/20 Revisado:26/01/21 Aceptado:24/04/21 Publicado:30/06/21

\begin{abstract}
Resumen
Introducción: La suspensión temporal de la docencia presencial, a raíz de la COVID-19 ha impulsado la transformación urgente de los procesos de enseñanza-aprendizaje en el contexto universitario, y la adaptación de la programación. Objetivo: Destacar las consecuencias generadas por la COVID-19 y el confinamiento en el alumnado universitario, y describir las experiencias docentes en dos asignaturas y sus respectivas estrategias evaluativas en dos universidades catalanas. Método: Descripción de la experiencia en las adaptaciones de la docencia y la evaluación de dos asignaturas, a consecuencia de la pandemia, que han contribuido a la transformación de la docencia en el ámbito de la educación superior, flexibilizando y adaptándose a las necesidades del alumnado en el último semestre. Conclusión: Las modificaciones y adaptaciones en las metodologías docentes y los procedimientos de evaluación han contribuido al afrontamiento de la situación adversa, y la evaluación de los procesos de enseñanza-aprendizaje en un escenario de alta complejidad.
\end{abstract}

Palabras clave: adaptación del estudiante; competencias del docente; enseñanza superior; COVID-19.

\section{Higher education and COVID-19: methodological adaptation and online evaluation at two universities in Barcelona}

\footnotetext{
Abstract

Introduction: The temporary suspension of face-to-face teaching activity, as a result of the global pandemic caused by COVID-19, has implied the urgent transformation of the teaching-learning processes in the university context, and an adaptation of programming. Goal: To highlight the consequences generated by COVID-19 and the confinement of university students, and to describe the teaching experiences in two subjects and their respective evaluation strategies in two Catalan
} 
universities. Method: Description of the experience in adapting the teaching and assessment of two subjects as a result of the pandemic, which have contributed to the transformation of teaching in the field of higher education, making it more flexible and adapting it to the needs of students in the last semester. Conclusion: The modifications and adaptations in the teaching methodologies and assessment procedures have contributed to the coping with the adverse situation, and the assessment of the teaching-learning processes in a highly complex scenario.

Key words: student adjustment; teachers qualifications; higher education; COVID-19.

\section{Educação superior e COVID-19: adaptação metodológica e avaliação on-line em duas universidades de Barcelona}

\section{Resumo}

Introdução: A suspensão temporária do ensino presencial como resultado da COVID-19 provocou a transformação urgente dos processos de ensino-aprendizagem no contexto universitário, e a adaptação da programação. Objetivo: Destacar as consequências geradas pela COVID-19 e o confinamento dos estudantes universitários, e descrever as experiências de ensino em duas disciplinas e as suas respetivas estratégias de avaliação em duas universidades catalãs. Método: Descrição da experiência nas adaptações do ensino e avaliação de duas disciplinas, como resultado da pandemia, que contribuíram para a transformação do ensino no domínio do ensino superior, tornando-o mais flexível e adaptando-se às necessidades dos estudantes no último semestre. Conclusão: As modificações e adaptações nas metodologias de ensino e procedimentos de avaliação contribuíram para o confronto da situação adversa, e a avaliação dos processos de ensino-aprendizagem num cenário altamente complexo.

Palavras-chave: adaptação do aluno; competências do professor; educação superior; COVID-19.

Ortega, D., Rodríguez, J., Mateos, A. (2021). Educación Superior y la COVID-19: Adaptación Metodológica y Evaluación Online en dos Universidades de Barcelona. Revista Digital de Investigación en Docencia Universitaria, 15(1), e1236. https:// doi.org/10.19083/ridu.2021.1275

\section{Introducción}

La aparición del COVID-19 en todo el mundo ha generado multitud de consecuencias que rebasan el ámbito laboral, sanitario, económico, social y académico (Nicola et al., 2020). A ello habría que añadirle las consecuencias individuales, con millones de personas obligadas a confinarse en sus hogares, viviendo una situación sin precedentes y a merced de las directrices de los diversos gobiernos, que han sido totalmente heterogéneas en todos los continentes, en función de la gravedad con la que ha impactado el virus y de la respuesta que puede otorgar el propio sistema de salud pública y comunitaria.

En el caso de España, el estado de alarma se decretó el 14 de marzo de 2020, con medidas de confinamiento que se incrementaron quince días después, paralizando toda actividad no esencial. Entre el amplio conjunto de medidas, se aplicó el cierre de todos los centros educativos (Zubillaga \& Gortazar, 2020).

En Cataluña ${ }^{1}$, las universidades suspendieron temporalmente su actividad docente de carácter

1 Es una de las 17 comunidades autónomas del Estado español, y que reconoce la Constitución española del año 1978. Su población es de 7,5 millones de persones, en una superficie de $32.108 \mathrm{~km} 2$. La capital es la ciudad de Barcelona, y el gobierno autónomo es la Generalitat. En el ámbito universitario, cuenta con un total de 12 universidades (entre públicas y privadas), donde se pueden cursar estudios oficiales de grado, postgrado y doctorado. 
presencial desde el 13 de marzo, a petición del gobierno de la Generalitat atendiendo las demandas del Consejo Asesor del Plan de Actuación del PROCICAT, un gabinete de crisis gubernamental. Esta decisión tenía una duración mínima de 14 días, y similares propuestas se dieron en la Comunidad de Madrid, País Vasco o La Rioja entre otras ${ }^{2}$.

En este contexto, los equipos docentes en enseñanza superior decidieron cambiar in extremis la metodología utilizada hasta la fecha. Todo ello ha supuesto, por un lado, poner de manifiesto las carencias en las competencias digitales del alumnado y del profesorado (Castellanos et al., 2017; Fernández et al., 2017) y, por otro lado, un reto para la flexibilización docente conforme a las necesidades del alumnado a medida que la pandemia evolucionaba. A esto se le sumaban dos motivos más. Así, diversas situaciones personales del alumnado han interferido en la actividad académica: las bajas por salud física, el cuidado de familiares a cargo y el aumento de horas de las actividades laborales consideradas esenciales. Hay que añadir también la brecha digital, pues una parte del alumnado ha necesitado beneficiarse de las ayudas tecnológicas que muchas universidades han implementado, ya que no todos contaban con los recursos y competencias digitales necesarias para las clases virtuales.

En ese sentido, hay que destacar que una pandemia con estas características ha generado múltiples consecuencias en el alumnado. Por lo tanto, hacer frente a las mismas, ha supuesto un desafío para el propio contexto universitario, y un abanico de nuevas oportunidades en torno a la creación o adaptación de metodologías docentes para el futuro, tal y como se verá a continuación.

Así pues, el artículo tiene por objetivo describir la experiencia docente en dos asignaturas de los grados de Educación Social y Pedagogía, de dos universidades catalanas, en el marco de la adaptación de contenidos curriculares y las estrategias evaluativas en un contexto de pandemia sanitaria y confinamiento.

2 Estas son otras de las 17 comunidades autónomas del Estado español El cierre de las universidades ha afectado a más de 200.000 estudiantes https://www.lavanguardia. com/politica/20200312/474097284624/educacio-ultima-orden-cierre-escuelas-catalunya.html

\section{Consecuencias generadas por la COVID-19 en el contexto universitario}

La aparición y desarrollo de la pandemia ha provocado una serie de consecuencias en la población general (Ministerio de Sanidad, Consumo y Bienestar Social, 2020), y de forma específica en el contexto universitario: la suspensión temporal de la actividad presencial docente en las aulas y aquellas actividades no consideradas esenciales (Giannini, 2020).

Ahora bien, la anulación de la presencialidad en el aula ha requerido una transformación y posterior transferencia de los planes docentes hacia una modalidad online in extremis, un reto tanto para los equipos docentes universitarios como para el alumnado, hacia un rediseño integral de las asignaturas pensadas y establecidas de forma inicial para ser impartidas presencialmente en el aula sin la necesaria planificación que requiere un nuevo formato de estas características.

Con todo, no puede obviarse, como se señalaba, el abanico de consecuencias que ha generado este fenómeno epidémico poliédrico en el conjunto de la población general (en la que se incluye alumnado y profesorado), destacando las siguientes:

- inicialmente, una de las mayores consecuencias se presenta en la salud física de las personas. En el momento de redactar este artículo, los casos confirmados en España ascendían a 2,467.520 personas, y el total de personas fallecidas se situaba en 28.325 (Ministerio de Sanidad, Consumo y Bienestar Social, 2020). Cataluña, con 60.754 personas afectadas y 5,666 defunciones, ha sido una de las áreas más castigadas, especialmente su zona central, ante esta grave situación las autoridades decidieron el aislamiento preventivo ${ }^{3}$ de pueblos y pequeñas ciudades incluso antes del confinamiento de la población general, por tanto, la cantidad de fallecidos y de contagiados ha generado un grave problema de salubridad

3 El Gobierno de Cataluña decreta el confinamiento de los municipios de Igualada, Vilanova del Camí, Santa Margarida de Montbui y Òdena. https://govern.cat/salapremsa/notes-premsa/383374/el-govern-decreta-el-confinament-dels-municipis-digualada-vilanova-del-cami-santa-margarida-de-montbui-odena 
social y económica. A ello habría que añadirse los pruebas e intervenciones quirúrgicas que se han suspendido al paralizarse el país mediante el estado de alarma, que ha durado 98 días,

- en el ámbito psicosocial y emocional, varias son las autoridades que han evaluado las consecuencias que la COVID-19 ha generado en el conjunto de la población (Arias et al., 2020; Ubillos \& González, 2020). Teniendo en cuenta que es una situación sin precedentes, impredecible e incontrolable, el contexto favorece la emergencia de ansiedad y problemáticas de salud mental posteriores (Chamarro, 2020; Mineka \& Kihlstrom, 1978). Un reciente estudio concluye que aumentó la sensación de incertidumbre y el malestar psicológico entre la población española en un $78 \%$ y un $46 \%$ respectivamente (Balluer$\mathrm{ka}$ et al., 2020). En este mismo estudio, se muestran indicadores de miedo o ansiedad, y cuya ontología del fenómeno se presume variada como las personas que han perdido su trabajo, el miedo a contagiarse del propio virus o las personas que han perdido un ser querido entre otras casuísticas. Y es que el miedo, la ansiedad y el estrés son elementos que han aparecido en numerosos estudios realizados en la población general, (Arias et al., 2020; Balluerka et al., 2020; Fernandes \& Ribeiro, 2020; Sandín et al., 2020; Vera-Villarroel, 2020) pero también en población universitaria (Islam et al., 2020; Rodriguez et al., 2020; Son et al., 2020; Unesco, 2020b; Wang \& Zhao, 2020),

- mención aparte merecen las condiciones relacionadas con las medidas de confinamiento. En cuanto a las condiciones en las que un individuo/núcleo familiar se ha sometido a las medidas de confinamiento, se han incrementado las desigualdades habitacionales dentro del contexto residencial de las personas. Cabe anotar que existen muchos factores que han contribuido en la calidad del confinamiento (Parrado-González \& León-Jariego, 2020), destacando: la superficie de la vivienda, la luz exterior, contar con espacios exteriores, o bien la propia compañía de las personas en la misma entre otras,
- finalmente, los estragos causados por esta pandemia global han tenido graves consecuencias en todos los ámbitos, como en el mercado laboral y en desigualdades económicas. Señala González (2020) que el mercado laboral puede ser una causa subyacente en el aumento de la desigualdad, afectando a la distribución general de los ingresos. La tendencia de destrucción de empleo genera las desigualdades en el mercado de trabajo y por extensión a las desigualdades de las familias si tenemos en cuenta que las rentas salariales representan la mayor parte de los ingresos familiares. Y es que la pérdida de los ingresos dentro de los colectivos perjudicados puede provocar, como sugiere Llorente (2020), un aumento del riesgo de pobreza y exclusión social, incrementando la desigualdad si el escenario no se acompaña de una adecuada política de sostenimiento del empleo.

Ahora bien, a las citadas consecuencias sufridas por el conjunto de la población, cabe añadir algunas específicas relacionadas con la suspensión temporal de la docencia presencial en las aulas y la posterior y necesaria adecuación metodológica a una nueva modalidad.

En primer lugar, el drama sin precedentes que ha representado el confinamiento para miles de alumnas y alumnos que han visto radicalmente alterados sus estilos de vida y sus rutinas diarias (Extremera, 2020), así como la pérdida en algunos casos de familiares a consecuencia del contagio; el asumir la atención y cuidado de familiares o la conciliación con la vida laboral en aquel alumnado que ha tenido que llevar a cabo tareas de teletrabajo o de trabajo presencial esencial.

$\mathrm{Y}$, en segundo lugar, reseñar los déficits en torno a los conocimientos informáticos y/o tecnológicos - tanto de docentes y alumnado. La pandemia ha puesto de manifiesto la ausencia de recursos y medios tecnológicos para paliar o reducir tales consecuencias generadas por la COVID-19 y su adaptación metodológica en la educación superior. Aquí es que donde se han evidenciado las consecuencias generadas por el COVID-19 en el contexto universitario y por extensión en la (re) adaptación hacia un modelo evaluativo de carácter digital y la necesidad de incrementar las com- 
petencias y herramientas digitales de la comunidad universitaria (Paredes-Chacín et al., 2020; Pozos \& Tejada, 2018). En este sentido, de acuerdo con varios autores, se hace necesario superar la brecha económica y la brecha digital (Matamala \& Hinostroza, 2020; UNESCO, 2020a).

Duart (2008) ya alertaba sobre la importancia de las Tecnologías de la Información y de la Comunicación (TIC) en la docencia universitaria, realzando el valor de la planificación previa para su posterior integración de manera que estas puedan contribuir al valor global de la institución. Y en similares parámetros Sevillano-García y Vázquez-Cano (2015) consideran que "aquellas organizaciones académicas que adopten innovaciones con TIC tendrán mucha más alta probabilidad de éxito si proveen y requieren formación específica sobre el uso adecuado de DMD [Digital Mobile Devices]" (p.115). En este sentido, cabe preguntarse si estos cambios no habrán venido para quedarse y supondrán además una modificación en la metodología docente de la enseñanza superior.

De igual manera advirtieron Galindo et al. (2017), quienes consideraban que en los próximos años en el sistema de educación superior urgía desarrollar competencias digitales para hacer frente a una extinción inevitable de empleos y profesiones que no estén enmarcadas dentro de este nuevo panorama. Consideraban que el propio sistema universitario requería eficaces mecanismos de alfabetización digital mediante un esquema de actualización permanente. Igualmente se pronunciaban Ocaña-Fernández et al. (2018), quienes asumían que las competencias digitales son requeridas en las aplicaciones de las TICs y las plataformas de interactividad en tiempo real.

Ahora bien, para una adaptación metodológica basada en la conectividad y en la capacidad de absorción de las herramientas digitales es necesario una igualdad de condiciones en los recursos existentes, para alcanzar los objetivos académicos con esta nueva modalidad. De esta manera, diversos autores alertan de las desigualdades que ha puesto de manifiesto la COVID-19 para poder proseguir de forma virtual el ritmo académico que requiere las nuevas condiciones virtuales, puesto que hay familias sin dispositivos en sus domicilios, otras que tienen que compartir los mismos entre todos sus miembros, o familias que no disponen de los recursos de conectividad a internet (Asuar, 2020; Echeita, 2020; Nogueira, 2020). Otras dificultades son las derivadas de conciliar la vida personal y laboral del profesorado.

En similares supuestos advierten Moreno-Rodríguez (2020), Zubillaga y Gortázar (2020), quienes consideran que la nueva situación ha magnificado cuatro brechas claramente identificables:

La brecha de acceso por la escasa universalización de la tenencia de dispositivos electrónicos y/o conectividad a internet.

La brecha de uso relacionada con la calidad y el tiempo de uso de dispositivos e Internet, puesto que existen hogares tal y como advierte el autor que a pesar de contar con los dispositivos estos son compartidos por los miembros de la unidad familiar.

La brecha de competencias, relacionada con las competencias digitales del profesorado y del alumnado para saber utilizar de forma óptima las plataformas digitales con fines educativos, así como la capacidad de creación de contenidos y acciones educativas a través de estas.

La autora y los autores del presente artículo añaden una cuarta: la brecha de género, que tiene que ver con las desigualdades que sufren históricamente las mujeres a la hora de acceder a diferentes profesiones y cargos de responsabilidad. Esto se ha puesto de manifiesto en muchas profesoras universitarias que han tenido que hacerse cargo de la atención de las hijas y los hijos, y de las tareas tradicionalmente asignadas en relación con el cuidado. Todo ello sin desatender a sus responsabilidades laborales, lo cual ha dificultado enormemente la conciliación familiar y laboral. Y es que la perspectiva de género es esencial en la respuesta a la COVID-19 en diferentes ámbitos, como señalan Castellanos-Torres et al. (2020), el Instituto de la Mujer (2020) y la Organización de la Naciones Unidas (ONU, 2020). En el marco socioeducativo en concreto, esta brecha continua vigente, tal y como apunta la UNESCO (2020a) y Juliá (2017).

De acuerdo con lo anterior, la pandemia por la COVID-19 ha generado en la comunidad universitaria incertidumbre y un desafío mayúsculo. A la decisión de proseguir con el ejercicio de la docencia, pese a las estrictas medidas de confinamiento y la nula presencialidad en las aulas, se sumó la ne- 
cesidad de una adaptación in extremis de los contenidos curriculares previamente establecidos. En este sentido, la COVID-19 ha supuesto un reto metodológico en la docencia y para la evaluación en las universidades españolas (Torrecillas, 2020), por el esfuerzo adaptativo que ha implicado.

A continuación, se detallarán las adaptaciones metodológicas elaboradas por la autora y los autores de este artículo que se han llevado tanto en la evaluación única y continuada, como en la adaptación de los procesos de enseñanza, aprendizaje online y el acompañamiento tutorial en las asignaturas El proceso de enseñanza-aprendizaje del grado de Educación Social, y la asignatura Intervención Educativa para la Inclusión Social, en el grado de Pedagogía. La importancia de estas adaptaciones tuvo como principal impacto, permitir al profesorado alcanzar los objetivos establecidos en las guías docentes de las asignaturas, a pesar de las restricciones generadas a consecuencia de la pandemia.

\section{Impacto de la COVID-19 en la docencia universitaria y adaptaciones metodológicas realizadas}

Para afrontar las consecuencias descritas con anterioridad, los rectorados de las universidades españolas generaron una serie de iniciativas para paliar desigualdades tecnológicas existentes, como la cesión de equipamientos informáticos y de conectividad a Internet del alumnado que así lo necesite. Algunas de estas iniciativas se recogen en el propio web de estas universidades ${ }^{4}$, continuando vigentes en el curso actual.

Del mismo modo, surgió la necesidad de una (re)adaptación tanto en parámetros de metodología docente, acompañamiento tutorial, procesos de enseñanza y aprendizaje, así como de las evidencias evaluativas. La (re)adaptación a la cual se hace referencia también vino marcada a través de las directrices estipuladas de forma gradual por las autoridades gubernamentales. Un ejemplo de ello se encuentra en una de las instrucciones elaboradas por el rectorado de una de las univer-

4 Pueden verse alguno de estos ejemplos en: https://www. ub.edu/web/ub/es/universitat/coronavirus/index.html; https://www.uab.cat/web/estudiar/grau/oferta-de-graus/ beques-d-estudi/detall-beca-1345798606891.html?param 1=1345825245167\&param2=UAB-FATWIRE sidades, que insta a los centros a habilitar un procedimiento específico para aquel alumnado con dificultades objetivas para hacer el seguimiento de la docencia virtual (situaciones laborales derivadas de la emergencia sanitaria, cuidado de terceras personas a su cargo, enfermedades, etc.).

Por su parte otra de las universidades también se pronunció al respecto mediante diferentes documentos, como: las directrices generales de funcionamiento de la docencia y la evaluación para el curso 2019-2020, aprobados por la Comisión Académica el 17 de abril de 2020; las directrices del rector de 28 de abril de 2020, en la que se detallan aportaciones encaminadas a modificar los criterios para la evaluación única y continuada; y finalmente la necesaria modificación y actualización de los procesos y metodologías de aprendizaje y tutorización del alumnado, que se trasladan y adaptan a cada una de las diferentes asignaturas del plan docente del grado.

En las primeras semanas de suspensión de la docencia en el aula, se puso de manifiesto la incertidumbre por la temporalidad del cese de la actividad presencial. De esta manera, las primeras consignas que buscaban los equipos docentes era evitar la desconexión académica a la par que social, mediante la planificación de actividadesfundamentalmente a través del campus virtual ya existente para mantener al alumnado conectado a la rutina académica habitual.

En este sentido es necesario manifestar el escaso margen de maniobra para la (re)planificación docente una vez decretada la suspensión total de la docencia presencial en el aula. Asimismo, existen algunos elementos que han facilitado-o dificultado- la adaptación de la docencia universitaria a un contexto virtual en plena pandemia ya que la docencia ha sido el área más afectada por la pandemia y el confinamiento, como señala García-Peñalvo (2020).

En primer lugar, las capacidades digitales necesarias para la transformación de la docencia presencial en una modalidad virtual. Las personas que tenían competencias digitales se han ajustado con una mayor facilidad a la adaptación metodológica, mientras que las personas que no reunían tales competencias sobrecargaron de manera significativa al alumnado con actividades, temas y lecturas complementarias, como advierte Moreno-Rodríguez (2020). El mismo autor sugiere 
que "se confundió la docencia online con un sistema 24/7 que suponía que, al permanecer todo el día en casa, los estudiantes disponían de más tiempo libre" (p. 2), y a su vez, estos interpretaron que el profesorado podía (o debía) estar disponible y accesible las 24 horas.

Otra de las dificultades halladas en los equipos docentes ha sido la propia actitud del alumnado hacia la adaptación tanto a la propia pandemia considerando su situación personal, como a la de las propias adaptaciones metodológicas al contexto universitario (Godoy et al., 2018). En este sentido y en consonancia con una mayor competencia digital, la actitud proactiva y proclive al cambio ha supuesto una mayor integración a las modificaciones metodológicas insertadas por los equipos docentes. Por el contrario, ha habido una amplia percepción de alumnado que no ha sabido o podido integrarse a la (re)adaptación metodológica, tanto por la escasez de recursos y competencias, como por las propias consecuencias del confinamiento de manera directa e indirecta (la asunción de nuevas responsabilidades por tener que atender a hermanas y hermanos, o hacerse cargo del cuidado de familiares con problemas de salud, etc.). En cualquier caso, la percepción en torno a la no asunción de contenidos puede generar determinadas consecuencias como la no superación de las asignaturas entre otras.

Otra de las cuestiones que suscitó controversia hace referencia a la flexibilidad en las normas de evaluación, así como el establecimiento de criterios mínimos que permitieran a los equipos docentes superar la improbable solución del aprobado general. Así, se han publicado interesantes orientaciones para la evaluación online como la propuesta por García-Peñalvo et al. (2020). Queda, no obstante, para un posterior análisis el impacto que puedan tener las modificaciones del proceso de evaluación en la vivencia del alumnado en relación con los mecanismos de evaluación de los aprendizajes.

Finalmente, y de forma paralela a las dificultades anteriormente mencionadas, la propia incertidumbre sobre la durabilidad de las medidas de confinamiento decretadas por las autoridades supuso un hándicap a la hora de planificar la actividad docente. Como se ha apuntado, la COVID-19 no ha supuesto una paralización temporal sino ha significado una (re)adaptación total de las materias, contenidos y metodología docente centradas cuasi en exclusividad en el carácter presencial de los procesos de enseñanza-aprendizaje.

Con todo, una vez se suspendió la actividad académica presencial en el aula de forma definitiva, y a sabiendas de que quedaban casi cuatro meses de docencia, se presentan las adaptaciones metodológicas para la continuidad de la docencia y la evaluación del segundo semestre en su modalidad o formato online realizadas en el grado de Educación Social y en el grado de Pedagogía en las asignaturas impartidas por la autora y los autores ("El proceso de enseñanza-aprendizaje" e "Intervención educativa para la inclusión social"). Estas adaptaciones recogen tanto el primer período de confinamiento (desde mediados de abril hasta las vacaciones de Semana Santa) como del segundo (desde Semana Santa hasta la finalización del período lectivo).

\section{Clases virtuales sincrónicas}

La adaptación virtual en el segundo semestre no podía obviar las clases magistrales. Para ello, se han utilizado metodologías para poder realizar las clases magistrales, adaptándolas al contexto virtual y respetando en lo posible el horario establecido en las guías docentes, siguiendo las directrices marcadas por la propia Universidad a tenor de los recursos tecnológicos que estas facilitaban, y teniendo en cuenta lo que se ha comentado con relación al impacto de la COVID-19 en el profesorado.

En el caso de una de las universidades, se ha ponderado el uso del Microsoft Teams para la realización de las clases magistrales de forma virtual, una plataforma de Microsoft que unifica y combina chats, reuniones de video, almacenamiento de archivos, la colaboración de archivos y la integración de las aplicaciones de la misma compañía. Dicha aplicación ha sido utilizada por los equipos docentes tanto para sus reuniones corporativas como para proseguir con la docencia a tenor de las posibilidades que permitía la misma.

Por su parte, desde la otra universidad, se ha utilizado la plataforma BBCollaborate, una herramienta de videoconferencia en tiempo real disponible para toda la comunidad educativa desde el campus virtual, y que facilita la realización de clases sincrónicas con el alumnado. Además, permite 
compartir la pantalla mientras el profesor lleva a cabo la explicación. Facilita también el intercambio de archivos de texto, sonido o fotografía. El profesorado puede organizar el aula virtual y la docencia creando grupos de alumnos para que resuelvan una tarea mientras con el otro grupo se puede realizar otra distinta.

Este procedimiento de clase magistral online supuso un intento de compensación de la no presencialidad en el aula, si bien, no todo el alumnado contaba con los medios técnicos necesarios para seguir con normalidad la docencia. Por otra parte, se puso de manifiesto las dificultades de interacción comunicativa dada la poca conectividad de gran parte del alumnado y la escasa participación activa (Leontyeva, 2018).

\section{Herramientas de e-Learning}

Las TIC han abierto un amplio repertorio de posibilidades didácticas para complementar la carga existente en torno a los contenidos curriculares en cualquier asignatura de enseñanza superior. El modelo docente -de forma mayoritaria- donde ejercen la docencia la autora y los autores del presente artículo se estipula a través de la presencialidad, si bien se considera que existen otros modelos que previa a la pandemia tenían un potencial desarrollo en este ámbito, como es el modelo de formación semipresencial: el e- Learning (Roig et al., 2013). No obstante, estas consideraciones tienen su relevancia en el nuevo escenario pospandemia (Pardo \& Cobo, 2020).

En este sentido y de forma complementaria a las clases virtuales sincrónicas, se han utilizado otras herramientas como las píldoras formativas elaboradas para explicar algún contenido específico de la materia y que puedan ser visualizadas de forma asíncrona y sin la necesidad de interacción con la otra parte (equipo docente). Así, se elaboraron diferentes cápsulas formativas en formato audiovisual de entre 5 y 12 minutos de duración a través del programa screencast matic. Dicho programa, en su versión gratuita, permite realizar vídeos de hasta un máximo de 15 minutos donde se comparte pantalla con el receptor del vídeo y donde se puede elaborar pequeños vídeos explicativos en formato mp4.

Esta herramienta permite visualizar las cápsulas formativas preparadas con anterioridad en función de la disponibilidad del alumnado, permitiendo además mantener vigente la interacción asincrónica con el alumnado.

\section{Tutorías online}

Sin lugar a duda, las tutorías grupales e individuales han sido una pieza esencial a la hora de abordar las principales adaptaciones metodológicas durante el segundo semestre. La tutoría sigue siendo "una función constante de toda actividad docente" (Lázaro, 1997, p. 233), una tarea ineludible en la formación de cualquier alumna o alumno universitario (Rodríguez et al., 2001). Se remarca que la frecuencia de esta estrategia metodológica se ha incrementado de forma exponencial, pues de forma genérica las dudas relativas a la propia materia, a los recursos presentados, o las pruebas de evaluación tales como las evidencias grupales o los exámenes siempre se han resuelto en el aula.

En contraposición, la mayoría del alumnado ha tenido una notable necesidad de tener un seguimiento docente mediante las tutorías, tanto para la resolución de dudas, como para sentirse acompañado y ayudado en la contención emocional a consecuencia de los cambios que ha generado la pandemia y el confinamiento en sus vidas. Y en este sentido, como señalan Rachtam y Firpo (2011), la tutoría virtual ha supuesto una especie de acercamiento hacia alumnado con dificultades para involucrarse en el proceso enseñanza-aprendizaje, ya sea por cuestiones personales o por no poder asistir presencial o sincrónicamente a la docencia como ha supuesto la propia pandemia. Y es que no hay que olvidar que la dimensión afectivo-emotiva es una de las dimensiones de la intervención tutorial también en la universidad (Rodriguez et al., 2001), contribuyendo al desarrollo integral del alumnado (Martínez et al., 2016).

\section{Lecturas complementarias}

Otra de las herramientas utilizadas, especialmente en el inicio de la suspensión de la docencia presencial en el aula, ha sido las lecturas complementarias, que permitían profundizar los contenidos dados en las clases virtuales o las cápsulas formativas anteriormente descritas, con el objetivo de incrementar el aprendizaje de una forma holística, integral y autónoma.

También se realizaron power points audiona- 
rrados $\mathrm{u}$ otros con contenido adicional de lo habitual para complementar el material docente.

A pesar del fácil acceso a estos materiales complementarios, surgió en alguna parte del alumnado la percepción de un aumento considerable de lecturas complementarias al resto del material curricular.

\section{Evidencias evaluativas}

Finalmente, no se puede obviar una de las finalidades de la docencia en enseñanza superior: la obtención de unos resultados académicos que suponen alcanzar los objetivos establecidos en los planes docentes. El propio Espacio Europeo Superior (EES) contempla una amplia variedad de evidencias evaluativas en su modalidad de evaluación continua y de acuerdo con la normativa que rige cada facultad.

En este sentido, la flexibilidad a la cual se aludía, en aras de garantizar la calidad del proceso de enseñanza-aprendizaje, también hace referencia a la evidencia evaluativa de cara a cursar las asignaturas y el éxito académico que el alumnado anhela. Así, en la docencia que se ha llevado a cabo en la asignatura Procesos de enseñanza y aprendizaje, una de las medidas ha sido la supresión de los exámenes tipo test y ponderar exámenes de carácter más reflexivo, con la posibilidad de generar respuestas de carácter abierto para poder demostrar la asunción de contenidos estipulados durante el temario a lo largo de la pandemia en la readaptación metodológica realizada en el presente curso.

Por su parte, en la docencia que se lleva a cabo en la asignatura Intervención Educativa para la Inclusión Social, y siguiendo las directrices del rectorado respecto de la evaluación, en concreto: se han flexibilizado de manera alternativa y realista los criterios de los requisitos para la evaluación única y la evaluación continuada, así como la supresión de exámenes tipo test en favor de un examen basado en un caso sobre el cual el alumnado tenía que reflexionar sobre los contenidos de la asignatura y, finalmente, la modificación del porcentaje de las evidencias evaluativas (resolución de dos casos prácticos, elaboración de un artículo de revisión bibliográfica en el ámbito de la intervención socioeducativa, y el examen final), a fin de garantizar el ajuste a unos criterios mínimos.

Estas modificaciones en las estrategias evaluativas han mejorado la percepción que el alumnado tenía en relación con la evaluación de las asignaturas, teniendo en cuenta la no presencialidad. Se considera que estas modificaciones no han disminuido la calidad de la atención y la valoración de los aprendizajes por parte del alumnado, a pesar de la falta de experiencia del mismo con este tipo de evaluaciones.

\section{Conclusiones}

Las adaptaciones metodológicas y las modificaciones de las estrategias evaluativas descritas han permitido a la autora y autores de este documento realizar un acompañamiento y seguimiento del alumnado durante el segundo semestre del curso 2019-2020. Del mismo modo, se ha podido alcanzar los objetivos preestablecidos en las guías docentes de las asignaturas donde se implementaron tales cambios. Todo ello en un contexto de alta incertidumbre en el conjunto de la comunidad universitaria, en el contexto de la propia evolución de la pandemia y sus consecuencias derivadas, y que generó un escenario académico de complejidad e impredecibilidad.

El principal hallazgo de este artículo, referido a las experiencias docentes descritas es la aportación de un amplio abanico de estrategias y adaptaciones metodológicas, que permitieron alcanzar los objetivos establecidos en los planes docentes y finalizar el curso con una cierta normalidad. Inexorablemente todo ello ha supuesto un desafío a fin de desarrollar la readaptación constante de las propias materias cursadas, y los contenidos dotados, la metodología docente y la forma de evaluar. Todo reto en la innovación educativa no está exento de la existencia de posibles limitaciones. En el estudio realizado, se hallan algunas limitaciones que abren vías futuras para continuar explorando y evaluando sobre las adaptaciones realizadas. Las adaptaciones se han valorado como positivas, en tanto que han permitido alcanzar los objetivos curriculares y las competencias descritas en los planes docentes de las asignaturas implicadas. Sin embargo, como línea de prospección futura, sería conveniente tener presente un estudio evaluativo de las adaptaciones realizadas para poder valorar de forma sistemática la idoneidad 
de estas para otros escenarios de la educación superior. Este estudio más exhaustivo del impacto y transferencia de las adaptaciones a otros contextos, no se ha podido llevar a cabo, pero se plantea como nuevo reto dada la posibilidad de continuar en un contexto de formación online en una docencia universitaria diseñada para llevarse a cabo de forma presencial.

Sin embargo, las adaptaciones metodológicas descritas a lo largo de este artículo pueden cobrar especial relevancia y utilidad por la posibilidad de que la COVID-19 recrudezca (en tanto aumente el número de contagiados y puedan regresar las estrictas medidas de confinamiento vividas durante los meses de marzo y abril del 2020). Estas experiencias permiten, además, intentar dar respuesta a las necesidades del alumnado que ha visto modificada su actividad académica a consecuencia de cambios laborales o personales derivados de la propia pandemia.

No obstante, ello requiere una modificación en la planificación docente de la metodología y de los recursos establecidos, pero también en lo que respecta a la normativa legal en materia de protección de datos. Un mayor uso de los medios tecnológicos ante una hipotética transformación de los procesos de enseñanza-aprendizaje requiere un trabajo previo de equipos multidisciplinares tanto de especialistas en seguridad de la información como de las redes, programadores etc., más allá del ejercicio docente. Del mismo modo requiere la formación específica de los docentes a fin de mejorar las competencias digitales para diversificar las estrategias evaluativas que se venían realizando hasta el inicio de la pandemia, ya que existe una demanda de las soluciones tecnológicas que permitan supervisar la evaluación de forma online (Fluck, 2019; Pathak, 2016).

Por otro lado, dicho desafío supone también una oportunidad para intentar reducir y/o paliar las desigualdades en torno a las brechas y competencias digitales anteriormente descritas. Las necesidades de adaptación metodológica de la docencia y la perentoria necesidad del uso de las TIC han respondido a una lógica conjunta del colectivo docente. Ello supone un cierto avance en contraposición a resultados hallados en investigaciones previas, donde su uso quedaba supeditado a la motivación y predisposición individual de los docentes (Gómez, 2016; Mercader \& Gairín, 2017; Torrecillas, 2020).

Se considera, por tanto, como apuntan $\mathrm{Mu}$ ñoz y Lluch (2020) y Pardo y Cobo (2020), que el momento de incertidumbre y excepcionalidad vivido sirva para desarrollar los conceptos de enseñanza-aprendizaje y ampliar las fronteras de la docencia más allá de un modelo tradicional de presencialidad en el ámbito de educación superior. Ello ha de permitir, en definitiva, el desarrollo de soluciones innovadoras para la educación inclusiva que se pretende como equipos docentes.

Además, se plantean algunas cuestiones para la reflexión ante un futuro a corto plazo:

Estos cambios que la situación de la pandemia ha generado ¿han llegado para quedarse?, ¿suponen una nueva forma de docencia universitaria con presencialidad y virtualidad?

En el curso 2020-2021, y teniendo en cuenta el riesgo de un posible rebrote ¿cómo se afronta el período lectivo? ¿Qué significa apostar por un escenario de docencia mixta sincrónica y asincrónica? ¿Cómo se adaptarán las evidencias evaluativas en este escenario en el que el aprendizaje autónomo cobra mayor protagonismo? ¿Cómo se resolverá el acompañamiento al alumnado en este escenario?

El profesorado y el alumnado que se ha visto también afectado por la pandemia y sus derivados, ¿podrá salir fortalecido de esta adversidad y afrontar de manera resiliente las diferentes brechas? ¿Se producirán cambios que permitan afrontar nuevos escenarios y dificultades? ¿Qué formación se presenta necesaria para afrontar la presencialidad mixta?

El contexto vivido, ¿permitirá modificar las políticas de las administraciones universitarias para favorecer la reducción de las desigualdades en torno a la brecha social, económica y digital del alumnado?

¿Se favorecerá la implicación del alumnado (student engagement) a fin de permitir un mayor protagonismo de este en su propio proceso de aprendizaje? ¿Cómo contribuirá el alumnado a la evaluación continua de estos aprendizajes?

En síntesis, las experiencias docentes descritas a lo largo del presente artículo muestran la conveniencia de que los equipos docentes aún en ciertas competencias tales como la flexibilidad, la 
motivación al cambio y un mayor conocimiento en torno a las TIC. Ello permite una mayor diversificación sobre las estrategias evaluativas que contienen los propios planes docentes, incrementando a su vez las metodologías e instrumentos utilizados en los procesos de aprendizaje en el contexto de educación superior.

\section{Referencias}

Arias, Y., Herrero, Y., Cabrera, Y., Chibás, D., \& García, Y. (2020). Manifestaciones psicológicas frente a la situación epidemiológica causada por la COVID-19. Revista Habanera De Ciencias Médicas, 19, e3350. http:// www.revhabanera.sld.cu/index.php/rhab/article/ view/3350http://www.revhabanera.sld.cu/index. php/rhab/article/view/3350

Asuar, B. (18 de marzo de 2020). Las desigualdades sociales se acentúan en la cuarentena educativa. Diario Público. https://cutt.ly/ktxdWd8https://cutt.ly/ktxdWd8

Balluerka, N., Gomez, J., Hidalgo, Mª ., Gorostiaga, A., Espada, J., Padilla, J., \& Santed, M. (2020). Las consecuencias psicológicas de la COVID-19 y el confinamiento. Informe de investigación. https://www.ub.edu/web/ ub/ca/menu_eines/noticies/docs/Consecuencias_ psicologicas_COVID-19.pdf

Castellanos, A., Sánchez, C., \& Calderero, J. F. (2017). Nuevos modelos tecnopedagógicos. Competencia digital de los alumnos universitarios. Revista Electrónica de In vestigación Educativa, 19(1). https://redie.uabc.mx/ redie/article/view/1148/1502

Castellanos-Torres, E., Tomás, J., \& Chilet-Rosell, E. (2020). COVID-19 en clave de género. Gaceta Sanitaria. Advance online publication. https://doi.org/10.1016/j. gaceta.2020.04.007

Chamarro, A. (2020). Impacto psicosocial del COVID-19: algunas evidencias, muchas dudas por resolver. Aloma: Revista de Psicologia, Ciències de l'Educació i de l'Esport, 38(1). http://www.revistaaloma.net/index.php/ aloma/article/view/432/200200237

Duart, J. M (2008). The Value of ICT in Higher Education. Revista de Universidad y Sociedad del Conocimiento, 5(1). https://www.redalyc.org/articulo. oa?id=7801120300 1

Echeita, G. (2020). La Pandemia del Covid-19. ¿Una Oportunidad para Pensar en Cómo Hacer más Inclusivos Nuestros Sistemas Educativos? Revista Internacio- nal de Educación para la Justicia Social, 9(1). https:// revistas.uam.es/riejs/article/view/12152

Extremera, N. (2020). Coping with the stress caused by the COVID-19 pandemic: future research agenda based on emotional intelligence. International Journal of Social Psychology, 35(3), 631-638. http://doi.org/10.10 80/02134748.2020.1783857

Fernandes, M. A., \& Ribeiro, A. A. (2020). Salud mental y estrés ocupacional en trabajadores de la salud a la primera línea de la pandemia de COVID-19. Revista Cuidarte, 11(2), e1222. https://doi.org/10.15649/cuidarte.1222

Fernández, E., Leiva-Olivencia, J. J., \& López-Meneses, E. (2017). Competencias digitales en docentes de Educación Superior. Revista Digital de Investigación en Docencia Universitaria, 12(1). https://revistas.upc. edu.pe/index.php/docencia/article/view/558/752

Fluck, A. E. (2019). An International review of exam technologies and impact. Computers \& Education, 132, 1-15. https://doi.org/10.1016/j.compedu.2018.12.008

Galindo, F., Ruiz, S., \& Ruiz, F. (2017). Competencias digitales ante la irrupción de la Cuarta Revolución Industrial. Estudos em Comunicação, 25(1). http://ojs.labcom-ifp.ubi.pt/index.php/ec/article/view/277

García-Peñalvo, F. (2020). La metodología antes que la tecnología para afrontar la nueva normalidad docente en la universidad [Webinar]. Universidad de Salamanca. https://bit.ly/2AOG1Rx

García-Peñalvo, F., Corell, A., Abella-García, V., \& Grande, M. (2020). La evaluación online en la educación superior en tiempos de la COVID-19. Education in the Knowledge Society, 21. https://doi.org/10.14201/eks.23013

Giannini, S. (2020). Covid-19 y educación superior: De los efectos inmediatos al día después. Revista Latinoamericana de Educación Comparada, 11(17), 1-57.

Godoy, V. H., Morales, K. F., \& Pulido, J. E. (2018). La actitud hacia la educación en línea en estudiantes. Revista de Investigación Educativa, 2(36), 349-364.

Gómez, J. (ed.) (2016). UNIVERSITIC 2016. Análisis de las TIC en las Universidades Españolas. Crue Universidades Españolas.

González, I. (2020). La profundización de la desigualdad por la COVID-19. En A. García (Ed.), La pandemia del COVID-19. Una visión interdisciplinar (pp. 14-21). Papeles de Discusión, Instituto Universitario de Investigación en Estudios Latinoamericanos-Universidad de Alcalá.

Gortázar,L., Moreno,J.M., \& Zubillaga,A. (2020). COVID y Educación II : Escuela en casa y desigualdad. Fundación Cotec para la innovación. https://online.flipping- 
book.com/view/350164/20/

Instituto de la Mujer (2020). La perspectiva de género, esencial en la respuesta a la COVID-19. Ministerio de Igualdad. http://www.inmujer.gob.es/diseno/novedades/ IMPACTO_DE_GENERO_DEL_COVID_19_(uv).pdf

Islam, M.A. Barna, S.D., Raihan, H., Khan, M. N. A., \& Hossain, M. T. (2020). Depression and anxiety among university students during the COVID-19 pandemic in Bangladesh: A web-based cross-sectional survey. PLOS ONE, 15(8), e0238162. https://doi.org/10.1371/journal. pone.0238162

Juliá, A. (2017). Género, nuevos riesgos sociales y desigualdad educativa. Brecha en el logro educativo de varones y mujeres [tesis doctoral]. Universidad de Barcelona. https://www.tesisenred.net/handle/10803/441747\#page=1

Lázaro, A. (1997). La acción tutorial de la función docente universitaria. Revista Complutense de Educación, 8(1), 233-252. https://dialnet.unirioja.es/servlet/articulo?codigo $=150210$

Leontyeva, I. A. (2018). Modern Distance Learning Technologies in Higuer Education: Introduction Problems. Eurasia Journal of Mathematics, Science and Technology Education, 14(10), em1578. https://doi. org/10.29333/ejmste/92284

Llorente, R. (2020). Impacto del COVID-19 en el mercado de trabajo: un análisis de los colectivos vulnerables. Documentos de trabajo. IAES-Instituto Universitario de Análisis Económico y Social, Universidad de Alcalá, (2). http://hdl.handle.net/10017/42247

Martínez, P., Martínez, M., \& Pérez, J. (2016). ¿Cómo avanzar en la tutoría universitaria? Estrategias de acción: los estudiantes tienen la palabra. REOP, 27(2), 80-98. https://doi.org/10.5944/reop.vol.27.num.2.2016.17115

Matamala, C., \& Hinostroza, J. E. (2020). Factores relacionados con el uso académico de Internet en educación superior. Pensamiento educativo, 57(1), 1-19. https:// dx.doi.org/10.7764/pel.57.1.2020.7

Mercader, C., \& Gairín, J. (2017). ¿Cómo utiliza el profesorado universitario las tecnologías digitales en sus aulas? REDU. Revista de Docencia Universitaria, 15(2). https://polipapers.upv.es/index.php/REDU/article/ download/7635/9191

Mineka, S., \& Kihlstrom, J. (1978). Unpredictable and uncontrollable events: a new perspective on experimental neurosis. Journal of Abnormal Psychology, 87(2), 256-271. https://doi.org/10.1037/0021-843X.87.2.256

Ministerio de Sanidad, Consumo y Bienestar Social (2020). Actualización $n^{\circ}$ 150. Enfermedad por el coronavirus (COVID-19). 28.06.2020. Situación en España. https://
www.mscbs.gob.es/profesionales/saludPublica/ccayes/alertasActual/nCov-China/documentos/Actualizacion_150_COVID-19.pdf

Moreno-Rodríguez, R. (2020) Reflexiones en torno al Impacto del Covid-19 sobre la Educación Universitaria: Aspectos a Considerar acerca de los Estudiantes con Discapacidad. Revista Internacional de Educación para la Justicia Social, 9(3). https://revistas.uam.es/ riejs/article/view/12227

Muñoz, J. L. \& Lluch, L. (2020). Educación y Covid-19: Colaboración de las Familias y Tareas Escolares. Revista Internacional de Educación para la Justicia Social, 9(3). https://revistas.uam.es/riejs/article/view/12182

Naciones Unidas (2020). Igualdad de género en tiempos del COVID-19. https://www.un.org/es/coronavirus/articles/igualdad-genero-covid-19

Nicola, M., Alsafi, Z., Sohrabi, C., Kerwan, A., Al-Jabir, A., Iosifidis, C., Agha, M., \& Agha, R. (2020). The socio-economic implications of the coronavirus pandemic (COVID-19): A review. International Journal of Surgery, 78, 185-193. https://doi.org/10.1016/j.ijsu.2020.04.018

Nogueria, R. (12 de marzo de 2020). El coronavirus agranda la brecha educativa. Ethic. Recuperado de https://cutt. ly/8txdvHJ

Ocaña-Fernández, Y., Valenzuela-Fernández, L., \& Garro-Aburto, L. (2019). Inteligencia artificial y sus implicaciones en la educación superior. Propósitos y Representaciones, 7(2), 536 - 568. http://dx.doi. org/10.20511/pyr2019.v7n2.274

Paredes-Chacín, A. J., Inciarte, A., \& Walles-Peñaloza, D. (2020). Educación superior e investigación en Latinoamérica: Transición al uso de tecnologías digitales por Covid-19. Revista de ciencias sociales, 26(3), 98-117.

Pardo, H., \& Cobo, C. (2020). Expandir la universidad más allá de la enseñanza remota de emergencia Ideas hacia un modelo híbrido post-pandemia. Outliers School.

Parrado-González, A., \& León-Jariego, J. C. (2020). COVID-19. Factores asociados al malestar emocional y morbilidad psíquica en población española. Revista Española de Salud Pública, 94(8). https://www.mscbs. gob.es/biblioPublic/publicaciones/recursos_propios/resp/revista_cdrom/VOL94/ORIGINALES/ RS94C_202006058.pdf

Pathak, B. K. (2016). Emerging online educational models and the transformation of traditional universities. Electronic Markets, 26(4), 315-321. https://doi. org/10.1007/s12525-016-0223-4

Pozos, K. V., \& Tejada, J. (2018). Competencias digitales do- 
centes en educación superior: niveles de dominio y necesidades formativas. Revista Digital de Investigación en Docencia Universitaria, 12(2), 59-87. http:// dx.doi.org/10.19083/ridu.2018.712

Quezada-Scholz, V. (2020). Miedo y psicopatología: La amenaza que oculta el COVID-19. Cuadernos de Neuropsicología / Panamerican Journal of Neuropsychology, 14(1). http://www.cnps.cl/index.php/cnps/article/ view/394

Rachtam, P., \& Firpo, D. (2011). Using Social Networking Technology to Enhance Learning in Higher Education: A Case Study using Facebook. Proceedings of the 44th Hawaii International Conference on System Sciences, 1-10. https://dx.doi.org/10.1109/HICSS.2011.479

Rodríguez, S., Dorio, I., Figuera, P., Fita, E., Forner, A., Mauri, T., Noguer, M., Homar, J. M., Torrado, M., \& Álvarez. M. (2001). Tutoría universitaria: una guía práctica. Universidad de Barcelona. http://diposit.ub.edu/dspace/handle/2445/1122

Rodriguez, Y., Solórzano, A., Vera, M. M., \& Parrales, M. L. (2020). Actitud frente a la distancia socio-afectiva de los estudiantes en la enseñanza virtual. UNESUM-Ciencias. Revista Científica Multidisciplinaria, 5(1), 57-74. http://revistas.unesum.edu.ec/index.php/ unesumciencias/article/view/313

Roig, R., Mengual, S., \& Rodríguez, C. (2013). Internet como medio de información, comunicación y aprendizaje. En J. Barroso y J. Cabero (Eds.), Nuevos escenarios digitales. Las tecnologías de la información y la comunicación aplicadas a la formación y desarrollo curricular (pp. 209-234). Pirámide.

Sandín, B., Valiente, R., García-Escalera, J. \& Chorot, P. (2020) Impacto psicológico de la pandemia de COVID-19: Efectos negativos y positivos en población española asociados al periodo de confinamiento nacional. Revista de Psicopatología y Psicología Clínica, 25(1). http://revistas.uned.es/index.php/RPPC/article/ view/27569/pdf

Sevillano-García, M. L., \& Vázquez-Cano, E. (2015). The Im- pact of Digital Mobile Devices in Higher Education. Educational Technology \& Society, 18(1). https://eric. ed.gov/?id=EJ1062522

Son, C., Hedge, S., Smith, A., Wang, X., \& Sasangohar, F. (2020). Effects of COVID-10 on College Sutdent's Mental Health in the United States: Interview Survey Study. Journal of Medical Internet Research, 22(9), e21279. https://doi.org/10.2196/21279

Torrecillas, C. (2020). El reto de la docencia online para las universidades públicas españolas ante la pandemia del COVID-19. ICEI Papers, 16. https://eprints.ucm. es/60050/1/16-1304.pdf

Ubillos, S., \& González, J.(2020). Afrontando el impacto del COVID-19. Resultados preliminares II. Universidad de Burgos. https://riubu.ubu.es/bitstream/handle/10259/5302/Afrontando_el_impacto_del_coVID19-2.pdf?sequence=1

UNESCO (2020a). La brecha de género en la matriculación en la escuela primaria se redujo a la mitad en los últimos 25 años. https://es.unesco.org/news/brecha-genero-matriculacion-escuela-primaria-se-redujo-mitad-ultimos-25-anos

UNESCO (2020b). Las universidades abordan el impacto del COVID-19 en los estudiantes desfavorecidos. https:// es.unesco.org/news/universidades-abordan-impacto-covid-19-estudiantes-desfavorecidos

Vera-Villarroel, P. (2020). Psicología y COVID-19: Un análisis desde los procesos psicológicos básicos. Cuadernos de Neuropsicología / Panamerican Journal of Neuropsychology, 14(1), 19-23. https://dialnet.unirioja.es/ servlet/articulo?codigo $=7365555$

Wang, C., \& Zhao, H. (2020) The impact of COVID-19 on anxiety in Chinese university students. Frontiers in psychology, 11, 1168. https://doi.org/10.3389/ fpsyg.2020.01168

Zubillaga, A., \& Gortazar, L. (2020). COVID-19 y educación: Problemas, respuestas y escenarios. Madrid, España: Fundación Cotec para la Innovación. https://online. flippingbook.co view/967738/

RIDU / Revista Digital de Investigación en Docencia Universitaria / e-ISSN: 2223-2516

(c) Los autores. Este artículo es publicado por la Revista Digital de Investigación en Docencia Universitaria del Área de Institutional Research and Effectiveness de la Dirección de Aseguramiento de la Calidad, Universidad Peruana de Ciencias Aplicadas. Este es un artículo de acceso abierto, distribuido bajo los términos de la LicenciaCreativeCommons Atribución 4.0 Internacional (CC BY 4.0) (https://creativecommons.org/licenses/by/4.0/deed.es), que permite el uso, distribución y reproducción en cualquier medio, siempre que la obra original sea debidamente citada." 